\title{
Compact 2G HTS power cable: new cold tests results
}

\author{
S S Fetisov ${ }^{1}$, V V Zubko ${ }^{1}$, S Yu Zanegin ${ }^{1}$, A A Nosov ${ }^{1}$ and V S Vysotsky ${ }^{1}$ \\ ${ }^{1}$ Russian Scientific R\&D Cable Institute (VNIIKP), Moscow, Russia, 111024
}

E-mail: vysotsky@ieee.org

\begin{abstract}
In Russian Scientific R\&D Cable Institute we continue studies to develop as compact as possible 2G HTS power cables for electric power systems. Compact cables should have minimized diameter compared to other co-axial HTS cables developed before. Recently we developed the cable prototype with four HTS layers in the core and two layers in the shield. Numerical optimization of compact cables has been used together with manufacturing technology to produce small diameter cables with uniform current distribution. The first cold test of the compact cable demonstrated that we were able to control the homogeneous current distributions in a compact cable with the inner diameter of the core $\sim 10 \mathrm{~mm}$ and the outer diameter of the shield $\sim 20 \mathrm{~mm}$. In this work we present more test results of this cable, including, mechanical test and high voltage tests. New test results demonstrated very high parameters of the compact cable developed. The compact cables perspectives for electric systems are discussed.
\end{abstract}

\section{Introduction}

Russian Scientific R\&D Cable Institute continue to perform researches and developments aimed at the maximum possible reduction in the mass and dimensions of power High Temperature Superconducting (HTS) cables. Compact HTS cables can be used in electric aircraft, ship electric propulsion systems or any other applications demanded small size and mass. Earlier, we reported [1] about manufactured and tested the prototype of a compact cable made of 2G HTS tapes. It has three layers in the cable core and two layers in the shield. During development of this cable, we were faced with the problem of obtaining a uniform distribution of currents between layers, due to the high sensitivity of compact cables to small manufacturing deviations. Ensuring a uniform distribution of current between the cable core layers, as well as between the shield layers in a multi-layer cable, is a complicated task for small diameter cables. The problem arises when thickness of cable layers become even as small as $~ 1-5 \%$ of a cable diameter.

In general, the main goal of our work is the justification of optimization methods, as well as the development of a production technology that provide a uniform distribution of current between the layers in a compact cable and low losses at alternating current.

The new calculation methods and optimized design of a compact cable made of 2G HTS tapes has been reported in [2]. The cable consisted of four layers in a cable core and two layers in a shield. The current distribution between the layers and the cable shield was optimized using two numerical models. The first model uses an electric circuit of a cable with current sources. The second, detailed threedimensional (3D) model uses the finite element method (ANSYS Emag). The influence of production deviations was also analyzed and taken into account [2].

The cable has been tested at liquid nitrogen and proper current distribution among layers has been confirmed [2]. AC losses have been measured as well. The possibility of obtaining a uniform current distribution in a compact cable with an inner diameter of the conductive core of $\sim 10 \mathrm{~mm}$ and an outer diameter of the shield of $\sim 20 \mathrm{~mm}$ was demonstrated.

In this paper we present the new experimental results of cold tests. In addition to results obtained before we performed mechanical and high voltage tests. During mechanical tests, the minimum bending diameters of the HTS cable were determined. The influence on the minimum bending diameter of the twist pitch of the tapes was obtained as well. High voltage tests permitted to evaluate the dielectric 
strength of the insulation used. New test results demonstrated very high parameters of the compact cable developed. The compact cables perspectives for electric systems are discussed also.

\section{Optimization, cable design and previous test results}

In this chapter we present briefly the results previously published in [2]. We consider it necessary for better understanding of the compact cable behaviour.

\subsection{Optimization results and influence of deviations in production}

For the cable prototype, 2G HTS tapes manufactured by SuperOx [3] with a total thickness of $0.105 \mathrm{~mm}$ was used. In order to reduce the polygonality of the layers in the core we used the HTS tapes with the $3 \mathrm{~mm}$ width. Average critical current of these tapes in the self-field at $77.4 \mathrm{~K}$ is $\sim 80 \mathrm{~A}$. For the shield we used the 2G HTS tapes with the $4 \mathrm{~mm}$ width. Their average critical current in self field at $77.4 \mathrm{~K}$ is $120 \mathrm{~A}$.

Next, an analysis was conducted of the sensitivity of the current distribution in a compact multilayer cable to the deviations of the diameters of the layers and the twist pitch of the HTS tapes in the layers during manufacturing [2]. In figure 1 we show the cases if radius of a layer has been slightly changed from the optimized value by the thickness of one tape that is $0.105 \mathrm{~mm}$. One can see that the manufacturing deviations in the diameters by the order of the thickness of the HTS tape has a large effect on the current distribution. Calculations show that even such a small deviation from the given values of the diameters of the layers can greatly disrupt the distribution of currents in the layers. Similar evaluations were done in [2] for deviations in twist pitches.

We concluded in [2] that manufacturing deviations in diameters has much more influence on current distribution than deviations in twist pitches. We also have to note that deviations in diameters of a shield does not affect the distribution of current in a core. Form these evaluations we could conclude in which layer the mistake in a diameter or in a twist pitch has more influence on the final current distribution.

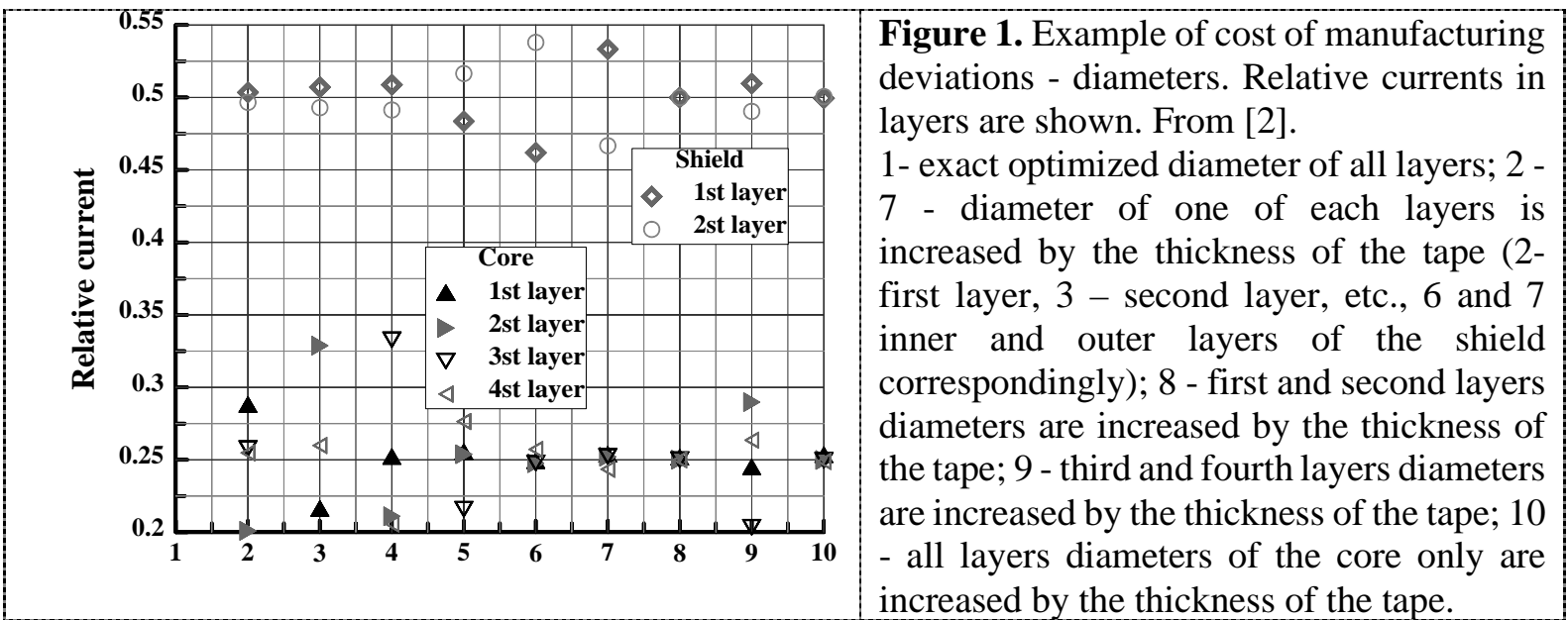

\subsection{The cable design}

To obtain a uniform current distribution in a compact multilayer cable, the precise winding of layers should be provided. During cable manufacturing, after winding of each layer, its average diameter was measured, then the parameters of the following layers were recalculated to be optimized. This method allows us to obtain a uniform current distribution in the prototype cable. It is also important to have a sufficiently rigid former and tightly wound insulation that do not change their diameters when HTS tapes are applied over them. 
The final optimized parameters of the core and the shield for our cable are listed in the Table 1 . To provide the minimal AC loss in the cable we have to have the gaps between tapes in cables layers as small as possible [4]. To achieve this, two tapes were added to second and third layers of the core and one tape was added to outer layer of the shield.

Table 1. Parameters of the model cable design [2].

\begin{tabular}{cccccc}
\hline $\begin{array}{c}\text { Number of } \\
\text { the } \\
\text { layer }\end{array}$ & $\begin{array}{c}\text { D min, } \\
\mathrm{mm}\end{array}$ & $\begin{array}{c}\text { Twist Pitch, } \\
\mathrm{mm}\end{array}$ & $\begin{array}{c}\text { Tape width, } \\
\mathrm{mm}\end{array}$ & $\begin{array}{c}\text { Number of } \\
\text { tapes }\end{array}$ & $\begin{array}{c}\text { Gap between } \\
\text { tapes, } \\
\mathrm{mm}\end{array}$ \\
\hline 1 & 10.32 & -56.2 & 3 & 9 & 0.15 \\
2 & 11.03 & -193.6 & 3 & 11 & 0.12 \\
3 & 12.03 & 94.3 & 3 & 11 & 0.21 \\
4 & 13.06 & 40.7 & 3 & 9 & 0.32 \\
5 & 18.25 & 349.4 & 4 & 13 & 0.36 \\
6 & 19.06 & -317.4 & 4 & 14 & 0.22
\end{tabular}

To adjust their diameters and to exclude direct current sharing between superconducting layers, all of them have been insulated by the Kapton ${ }^{\mathrm{TM}}$ tape with $20 \mathrm{~mm}$ width and $0.05 \mathrm{~mm}$ thickness as follows:

1. After the first superconducting layer - two Kapton ${ }^{\mathrm{TM}}$ layers with 50 percent overlap, one layer without overlap.

2. After the second superconducting layer, four Kapton ${ }^{\mathrm{TM}}$ layers with 50 percent overlap.

3. After the third superconducting layer, four Kapton ${ }^{\mathrm{TM}}$ layers with 50 percent overlap, one layer without overlap.

4. After the fourth superconducting layer, one Kapton ${ }^{\mathrm{TM}}$ layer with an overlap of 50 percent;

For the insulation between the cable core and the shield we used 12 layers of impregnated fiberglass tape. Tape width - $15 \mathrm{~mm}$, tape thickness $0.19 \pm 0.02 \mathrm{~mm}$. Each tape has been wound closely without overlapping, each subsequent layer with a 50 percent shift. The final insulation was made of two Kapton $^{\mathrm{TM}}$ layers, one without overlap and the second with 50 percent overlap. The total thickness of insulation between the cable core and the shield was $\sim 2.5 \mathrm{~mm}$.

The shield insulation was as follows:

5. After the first superconducting layer, three Kapton ${ }^{\mathrm{TM}}$ layers with 50 percent overlap.

6. After the second superconducting layer, one Kapton ${ }^{\mathrm{TM}}$ layer with 50 percent overlap.

\subsection{Current distribution and AC loss test results}

Test facility and instrumentation of the test bench in Russian Scientific R\&D Cable Institute have been presented in our previous works [5, 6]. The standard test program for any model of HTS cable usually includes:

- DC tests to determine critical currents in each layer;

- AC tests to determine the distribution of current between layers under alternating current conditions at frequencies of 50-400 Hz;

- Measurement of AC losses by electric method at frequencies of 50-400 Hz.

\subsubsection{Test results [2]}

The critical currents $\left(I_{c}\right)$ were determined by the criterion of $1 \mu \mathrm{V} / \mathrm{cm}$ for each layer at the temperature $77.4 \mathrm{~K}$. In the cable core, the following $I_{c}$ values were obtained: in the first (innermost) layer is $I_{c}=729$ $\mathrm{A}$; in the second layer $I_{c}=891 \mathrm{~A}$; in the third layer $I_{c}=895 \mathrm{~A}$; in the fourth layer $I_{c}=735 \mathrm{~A}$. In total in the cable core critical current is $3250 \mathrm{~A}$, which corresponds to the expected $I_{c} \sim 3200 \mathrm{~A}$. The critical currents on the shield were: in the internal layer $I_{c}=1560 \mathrm{~A}$; in the outer layer $I_{c}=1660$, in total $I_{c}=3220 \mathrm{~A}$, which is also very close to the expected $\sim 3240 \mathrm{~A}$. Thus, we can conclude that in our cable there is no degradation of the critical current in HTS tapes due to mechanical deformation during manufacturing (twisting of the tapes when they are applied on small diameter in the cable core). 


\subsubsection{AC Current Distribution Between Layers and AC Loss Measurements [2]}

The results of measurements of AC current distribution versus amplitude of total current were presented in [2]. The results of new measurements of AC current distribution for different frequencies at total current $2 \mathrm{kA}$ are shown in figure 2 . We made several measurements at different total currents, they all coincide very well. One can see, that as the result of optimization and manufacturing method used we were able to achieve practically uniform current distribution among cable layers. In the cable core uniformity is better than $10 \%$ and in the shield is better than $5 \%$. Slight deviation from uniformity most probably could be attributed to current leads influence [2].

We performed AC loss measurements in the model compact cable to compare them with those measured in 2G HTS cable models tested before. AC loss measurements were done by the electrical method described in [6]. In figure 3 the AC losses per cycle and per meter are shown for the cable core and the cable shield for different frequencies being recalculated per one tape. For comparison, the measured AC losses are shown in the shield of compact 2G HTS cables we produced and tested earlier [1]. AC losses in the shield are practically the same as in our first compact cable described in [1], while in the core AC loss are slightly less in this cable due to less width of the tapes. In any cases AC losses in 2G HTS cables are sufficiently less than in 1G HTS cables, for example described in [5]. AC losses per cycle does not depend on frequency like we found in [1] for our first compact cable.

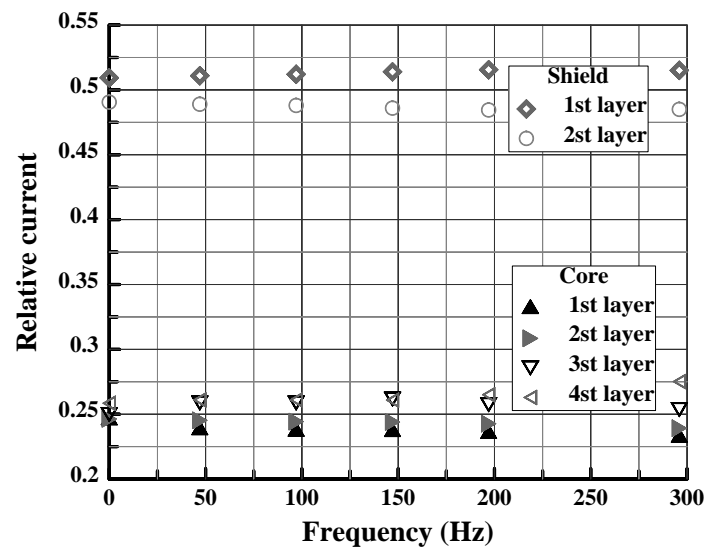

Figure 2. Measured AC current distribution among layers of the cable core and the shield of the HTS cable at different frequency.

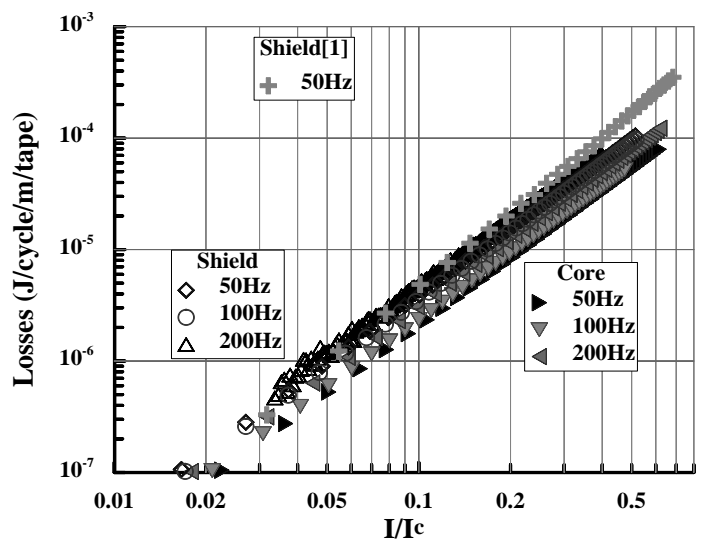

Figure 3. Comparison of AC losses per cycle and per tape versus relative current at different frequencies in layers of the compact cable model and in the shield of compact cables produced and tested in the Russian Cable Institute earlier (from [1]).

\section{Mechanical test}

To find out the effect of deformation on the critical current characteristics of the finished cable, we performed the tests whose main purpose is to determine the minimum allowable bending radius of the cable when laying, mounting, winding on technological and shipping drums. As a result of bending, the cable changes its current-carrying capacity. That is why, the value of the minimum bending diameter was introduced to describe the properties of HTS tapes in a cable. This is the diameter of bending at which the critical current drops not lower than 0.95 of the critical current in the initial state.

Initially, the critical current and the index $n$ of the $V-I$ characteristics were measured for each layer of the straight cable section after cooling in liquid nitrogen. Then the following actions were performed:

- warming to room temperature;

- bending of the cable around the mandrel of the certain diameter;

- straightening of the cable;

- cable cooling in liquid nitrogen;

- measurement of critical current $I_{c}$ and the index $n$. 
With each subsequent step, the radius of the mandrel gradually decreased, and the bending was carried out on a sample completely warmed up to room temperature, and always in the plane of the same meridional section of the cable. In this case, only the working section (measured length) was subjected to bending between the current leads, which were kept straight at all stages of the experiment (see figure 4).

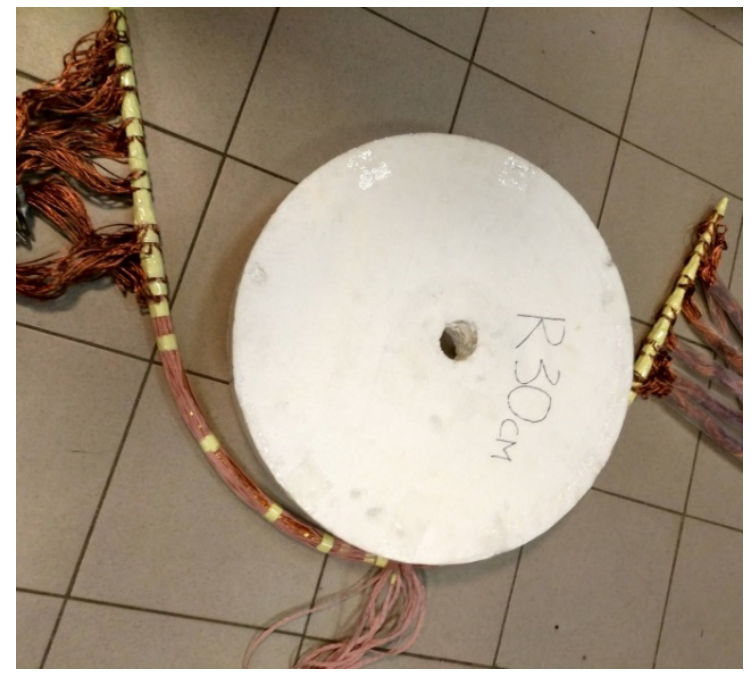

Figure 4. HTS cable during bending around the mandrel.

After processing the results, we obtained the dependences on the cable bending radius for each of the layers of the relative critical currents (figure 5) and the power law index $n$ (figure 6).

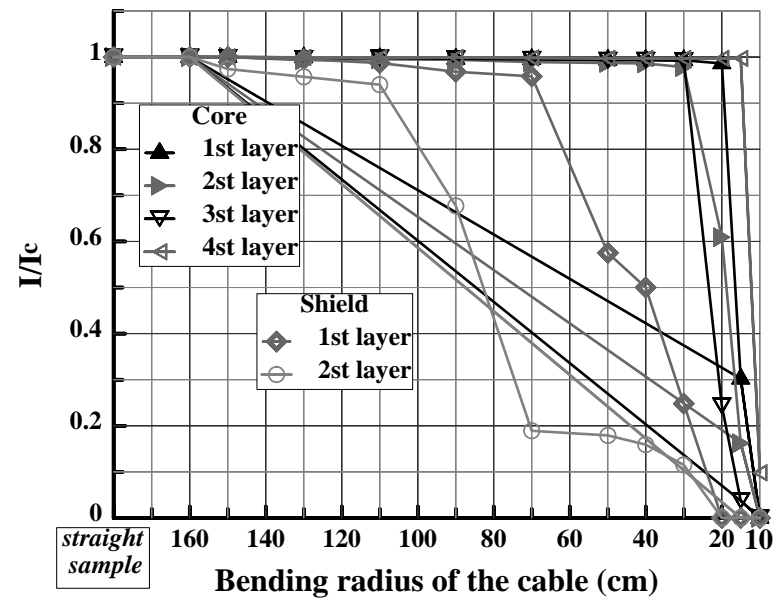

Figure 5. Dependences of relative critical currents in the cable layers (compared with the straight sample) on the cable bending radius.

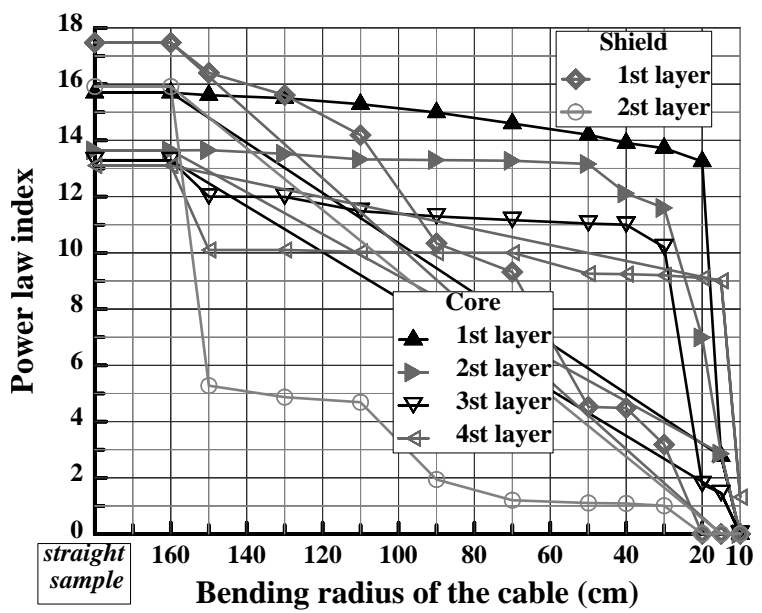

Figure 6. Dependences of the power law index $\mathrm{n}$ of the $V-I$ characteristic in the cable layers on the cable bending radius.

As a result, the influence of deformation on current characteristics of the HTS cable has been found. From the dependences presented one can see that the index $n$ degradation is noticeable first without a visible decrease in the critical current. With further deformation, the index $n$ continues to decrease and then the critical current degradation becomes noticeable. Thus, depending on the application of the cable, when determining the minimum bending diameter allowable, the index $n$ degradation should be taken into account. The minimum bending radiuses are presented in Table 2 for each layer of our cable, the data are taken from figure 5 for current degradation. 
Table 2. Minimum allowable bending radii for each layer of the compact HTS cable.

\begin{tabular}{lllc}
\hline \multicolumn{1}{c}{ Layer } & Layer diameter, $\mathrm{mm}$ & Twist pitch, $\mathrm{mm}$ & Minimum bending radii, mm \\
\hline Core, layer 1 & 10,3 & 56 & 200 \\
Core, layer 2 & 11 & 194 & 300 \\
Core, layer 3 & 12 & 94 & 300 \\
Core, layer 4 & 13,1 & 41 & 150 \\
Shield, layer 1 & 18,2 & 349 & 700 \\
Shield, layer 1 & 19 & 317 & 1100
\end{tabular}

Despite the absence of an explicit dependence, there is a correlation between the values of the twist pitch of the HTS tapes in the layer and its diameter with the minimum bending radius of the given layer. The minimum bending radius is directly proportional to the twist pitch of the HTS tapes and inversely proportional to the diameter of the layer. One can note, that the core layer 4 has smaller bending radii than core layer 2, because of shorter twist pitch and in spite of its larger diameter.

\section{High voltage electrical test}

The cable model described has electrical insulation with thickness $\sim 2.5 \mathrm{~mm}$. We decided to perform high voltage (HV) test to determine the potential of the compact cable presented as a power cable in some electrical power system.

At the ends of 2200 mm long sample the HV terminations were prepared. For this, at the end of the sample, insulation was thickened, a metal rings have been installed on the thickening, and superconducting tapes were wound on it that formed HV termination. The electric field strength in the $\mathrm{HV}$ terminations becomes less than in the cable insulation, where the dielectric strength is studied (figure 7 a).

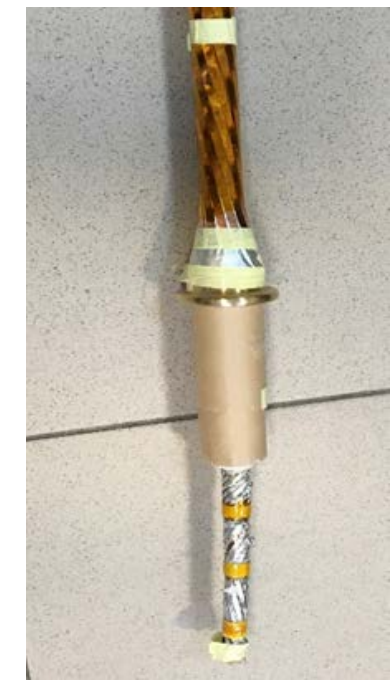

a

Figure 7. a - the HV termination prepared; b - the cable sample in liquid nitrogen bath for HV test

Tests were performed in the liquid nitrogen at atmospheric pressure (figure $7 \mathrm{~b}$ ). Voltage from a HV source was applied to the cable core. The cable shield has been grounded. The voltage was smoothly raised by steps at multiples of $5 \mathrm{kV}$ with an exposure at each step for 15 minutes. When the voltage has been risen above $20 \mathrm{kV}$ after exposure for $15 \mathrm{~min}$, the insulation has been broken. The HV test 
demonstrated that the cable successfully passed the tests up to $20 \mathrm{kV}$ DC, that means allowable operation level at least $10 \mathrm{kV}$ amplitude.

\section{Conclusions}

An optimized compact HTS power cable has been manufactured and extensively tested at Russian Scientific R\&D Cable Institute. The cable consists of the cable core having 4 layers of 2G HTS tapes with $3 \mathrm{~mm}$ width and the shield having two layers of 2G HTS tapes with $4 \mathrm{~mm}$ width. The main goal of the work was to develop and to justify optimization methods and production technology to meet the requirements for a uniform distribution of current between the layers and to obtain low AC losses. Using the developed numerical models, optimization of the compact cable was carried out and the influence on the current distribution of manufacturing deviations was analyzed. It was found that to achieve acceptable results, it is necessary to measure the actual outer diameter of each layer after its manufacture, and then make adjustments of the optimization parameters of the remaining layers, such as the tape spacing in the layer. It was demonstrated that with basic technologies and computing models developed, it is possible to control appropriately the applying the tapes into a layer to obtain a uniform current distribution in a compact cable with an inner core diameter of $\sim 10 \mathrm{~mm}$ and an outer diameter of the shield of $\sim 20 \mathrm{~mm}$. Measurements of AC losses in a compact cable showed that they are close or less to those in previously tested 2G HTS cables.

The influence of deformation on current characteristics has been studied. The main purpose was to determine the minimum allowable bending radius of the cable during laying, mounting, winding on technological and shipping drums. As the result of these studies, the influence of bending on the current characteristics of the HTS cable has been determined.

High voltage tests performed in liquid nitrogen at atmospheric pressure demonstrated dielectric strength of the insulation used as much as $20 \mathrm{kV}$ DC. This means allowable operation voltage $\sim 10 \mathrm{kV}$. If to consider the three-phase AC power line consisted of three our cables it will have $\sim 45 \mathrm{~mm}$ outer diameter and will be able to transfer at $3000 \mathrm{~A}$ about 50 MVA of electric power. This is very high level of power density transfer $p \sim 0.8 \cdot 10^{6} \mathrm{~W} / \mathrm{cm}^{2}$ that is by the order of value close to the high power hybrid energy transfer systems [7].

Our model cable is the most compact HTS power cable in the world with currents $\sim 3 \mathrm{kA}$ at liquid nitrogen temperature, produced and tested up to present time. This is the prototype for the compact and powerful energy transfer systems for electric aircrafts, ship electric propulsion systems or any other applications demanded small size and mass.

\section{Acknowledgment}

This work is supported by the Russian Scientific Foundation under Grant №16-19-10563П

\section{References}

[1] Fetisov S et al 2018 IEEE Trans. Appl. Supercond. 284

[2] Fetisov S, Zubko V, Zanegin S, Vysotsky V 2019 J. Phys: Conf. Ser. 502012179

[3] Available at http://www.superox.ru/en.

[4] Clem J and Malozemoff A 2010 Supercond. Science and Technology 233034014

[5] Vysotsky V et al 2011 IEEE Trans. Appl. Supercond, 213 1001-1004

[6] Fetisov S et al 2014 J. Phys: Conf. Ser. 5073032063

[7] Vysotsky V et al 2013 IEEE Trans. Appl. Supercond. 2335400906 